\title{
Infecciones cutáneas polimicrobianas que precisan colaboración multidisciplinar
}

\author{
Carlos Gómez Gonzáleza , Christian González Carrerasº Luz Belén Zambrano Centeno .
}

\author{
${ }^{a}$ MIR de tercer año de \\ Medicina Familiar y \\ Comunitaria. Centro de \\ Salud Talavera Centro. \\ Talavera de la Reina. Toledo \\ (España). \\ ${ }^{b}$ D.U.E. Centro de Salud de \\ Oropesa. Oropesa. Toledo \\ (España). \\ ${ }^{c}$ Especialista en Medicina \\ Familiar y Comunitaria. \\ Centro de Salud Puente \\ del Arzobispo. Puente del \\ Arzobispo. Toledo (España).
}

Correspondencia:

Carlos Gómez González.

Correo electrónico:

ccgg143@gmail.com

Recibido el 27 de septiembre de 2013.

Aceptado para su

publicación el 28 de enero de 2014.

\section{RESUMEN}

Paciente varón de 52 años que acude a la consulta por presentar prurito intenso en pie derecho de 15 días de evolución. Antecedente personales: hipercolesterolemia, hipertrigliceridemia y estenosis de uretra en tratamiento con dilataciones. Intervenido de prótesis de cadera por fractura y múltiples intervenciones quirúrgicas en ambos pies por fracturas producidas en accidente de tráfico. En la actualidad recibe tratamiento habitual con simvastatina y ácido omega tres. Es independiente para las actividades de la vida diaria y reside en un pueblo a $35 \mathrm{~km}$ del centro hospitalario más cercano. Exploración: Enrojecimiento de región dorsal y zona interdigital de $3^{\text {er }}$ a $5^{\circ}$ dedo de pie derecho, acompañado de maceración y sin presencia de onicomicosis en ninguno de los dedos. Pruebas complementarias: se extraen cultivo de lesiones ulcerosas donde se objetivan infección por Pseudomonas aeruginosa, Sthapylococcus aureus meticilin resistente y Enterococcus faecalis. Diagnóstico diferencial: intértrigo, tinea pedis, psoriasis inversa, eritrasma, candidiasis. Discusión: Las infecciones cutáneas son muy frecuentes en las consultas de atención primaria y el diagnóstico diferencial debe realizarse correctamente y con ayuda de las pruebas complementarias antes de iniciar tratamiento. La utilización de antibioterapia intravenosa de manera ambulatoria es una opción adecuada en estos casos.

Palabras clave: Enfermedades Cutáneas Infecciosas. Intertrigo. Atención Primaria de Salud.

\section{ABSTRACT}

Polymicrobial skin infections requiring a multidisciplinary approach

A male patient aged 52 visits the outpatient clinic referring intense itching in his right foot over 15 days. Personal history: hypercholesterolemia, hypertriglyceridemia and urethral stenosis under dilatation therapy. Hip replacement surgery due to fracture and multiple surgical interventions in both feet due to fractures occurred in a traffic accident. At present he is on permanent therapy with simvastatin plus omega-3 fatty-acid. He is independent for daily life activities and lives in a small town $35 \mathrm{~km}$ away from the closest hospital center. Physical examination: Redness in the dorsal area and interdigital area from the third to fifth toe in his right foot accompanied by maceration with no presence of toenail onychomycosis. Complementary tests: skin samples of ulcerous lesions were obtained for culture, showing infection with Pseudomonas aeruginosa, Methicillin resistant Staphylococcus aureus and Enterococcus faecalis. Differential diagnosis: Intertrigo, tinea pedis, inverse psoriasis, erytharsma, candidiasis. Discussion: Skin infections are very frequent in primary care and a correct differential diagnosis is necessary and the pertinent complementary tests should be carried out before starting therapy. The use of ambulatory intravenous antibiotic therapy can be an appropriate option in these cases.

Key words: Skin Diseases, Infectious. Intertrigo. Primary Health Care.

\section{INTRODUCCIÓN}

El intertrigo es una inflamación cutánea en la que, a pesar de su frecuencia en las consultas de Atención Primaria (AP), no hay datos sobre su prevalencia ni incidencia ${ }^{1}$. Se caracteriza por la aparición de lesiones eritematosas descamativas, con tendencia a la maceración en zonas de roce, como la región umbilical, espacios interdigitales, etc. ${ }^{2}$; y que puede sobreinfectarse por diferentes microorganismos: micóticos (Candida albicans), bacterianos (Pseudomonas auriginosa, principal causante de lesiones interdigitales ${ }^{2-4}$; Sthapylococcus aureus, Proteus mirabilis, etc. $\left.{ }^{2}\right)$, e incluso mixtos ${ }^{5}$. Hay una serie de factores que predisponen a esta patología, como algunas enfer- 
medades, falta de higiene y malnutrición ${ }^{4}$. El diagnóstico es clínico, aunque la utilización del estudio microbiológico mediante cultivo con antibiograma es una prueba a la cual hay acceso desde AP y que nos aportaría información importante para el correcto manejo. El tratamiento debe iniciarse corrigiendo aquellos factores predisponentes y evitando la humedad y la fricción de las zonas afectas, para lo cual en ocasiones pueden utilizarse agentes absorbentes, cremas de barrera y corticoides tópicos ${ }^{2}$. En caso de ausencia de respuesta al tratamiento o complicaciones tendríamos que pasar al tratamiento antibiótico o antifúngico dependiendo del agente causal².

En ocasiones, el tratamiento para las infecciones por Pseudomonas con antibiótico por vía oral no es suficiente ${ }^{6}$. Cuando se nos presenta esta situación debemos plantearnos las siguientes cuestiones: ¿es necesario el ingreso del paciente únicamente para la administración de antibióticos?; ¿hay otra forma de realizar dicho tratamiento?

Ya desde la invención en los años 50 de las cánulas de plástico hasta la actualidad, conocemos distintas experiencias de administración de tratamiento antibiótico de manera extrahospitalaria que han demostrado un ahorro importante, y sin registro de aumento en las complicaciones de los tratamientos $^{7}$. Aunque viene realizándose en EE.UU. desde 1974, cuando fueron descritos los primeros casos, en nuestro país fue en 1987 cuando encontramos la primera experiencia publicada sobre la administración ambulatoria de tratamiento antibiótico intravenoso $0^{7,8}$.

\section{OBSERVACIONES CLÍNICAS}

Paciente varón de 52 años con antecedentes de hipercolesterolemia e hipertrigliceridemia. Intervenido de prótesis de cadera y múltiples intervenciones quirúrgicas en ambos pies por fracturas producidas en accidente de tráfico. En la actualidad recibe tratamiento habitual con simvastatina y ácido omega tres. Es independiente para las actividades de la vida diaria y reside en un pueblo a $35 \mathrm{~km}$ del centro hospitalario más cercano. Acude a la consulta de AP por presentar prurito intenso y enrojecimiento de la región dorsal y zona interdigital de $3^{\text {er }}$ a $5^{\circ}$ dedo del pie derecho, acompañado de maceración y sin presencia de onicomicosis en ninguno de los dedos (figura 1).

Su médico de AP inició tratamiento con miconazol al $2 \%$ en crema que, al no mejorar, cambió por cloxacilina cada 4 horas y ácido fusídico cada 12 horas durante una semana. Al no encontrar tampoco mejoría fue remitido al Servicio de Dermatología, donde lo diagnosticaron de intertrigo e inició tratamiento con cefditoreno $400 \mathrm{mg}$ cada 24 horas y curas locales complementadas con pomada corticoidea. A los pocos días acude al Punto de Atención Continuada (PAC) aquejando empeoramiento de la clínica y alteración del estado general. A la exploración presenta petequias en la zona perilesional junto a autoeccematización y ausencia de pulso pedio y tibial posterior de dicha extremidad. Tras comentarlo con el facultativo de guardia el enfermero encargado de realizar la cura, se decidió remitir al paciente al centro hospitalario de referencia para valoración y extracción de muestra para cultivo.

Tras acudir al Servicio de Urgencias Hospitalario (S.U.H.) en varias ocasiones y a pesar de reajustes del tratamiento con antibiograma, las lesiones no mejoran. El dermatólogo inicia tratamiento antibiótico con ceftazidima $1 \mathrm{~g}$ cada 12 horas intravenosa durante 10 días, lo cual obliga al paciente a acudir al S.U.H. para administrarse dicha medicación. Debido a los problemas que supone realizar diariamente $170 \mathrm{~km}$ para la administración del fármaco, se pone en contacto el S.U.H con el PAC de la localidad del paciente, donde acceden a la administración del tratamiento intravenoso. Tras estas medidas adoptadas, el paciente presenta en 4 días una clara mejoría y al finalizar el tratamiento una total recuperación de la integridad cutánea y desaparición de signos locales de inflamación a los 15 días del inicio del tratamiento definitivo (figura 2).

\section{COMENTARIOS}

La apariencia clínica con la que cursan los intertrigos con sobreinfección fúngica y bacteriana es muy similar en cuanto a zona afectada y síntomas locales ${ }^{2,3}$. Es frecuente caer en el error de tratar los intertrigos como una infección micótica sin haber realizado previamente un cultivo, lo cual es importante para evitar dicha confusión. En el tratamiento de esta patología, además de la antibioterapia ajustada con cultivo y antibiograma, son muy importante las medidas higiénicas ${ }^{1,2}$. En nuestro caso, el paciente no realizaba de forma adecuada las medidas higiénicas y, además, se inició el tratamiento prematuramente, manteniéndose posteriormente de forma empírica a pesar de la mala evolución, hecho que prolongó el proceso. Probablemente este fue uno de los motivos por el cual precisó tratamiento antibiótico intravenoso. También nos encontrá- 

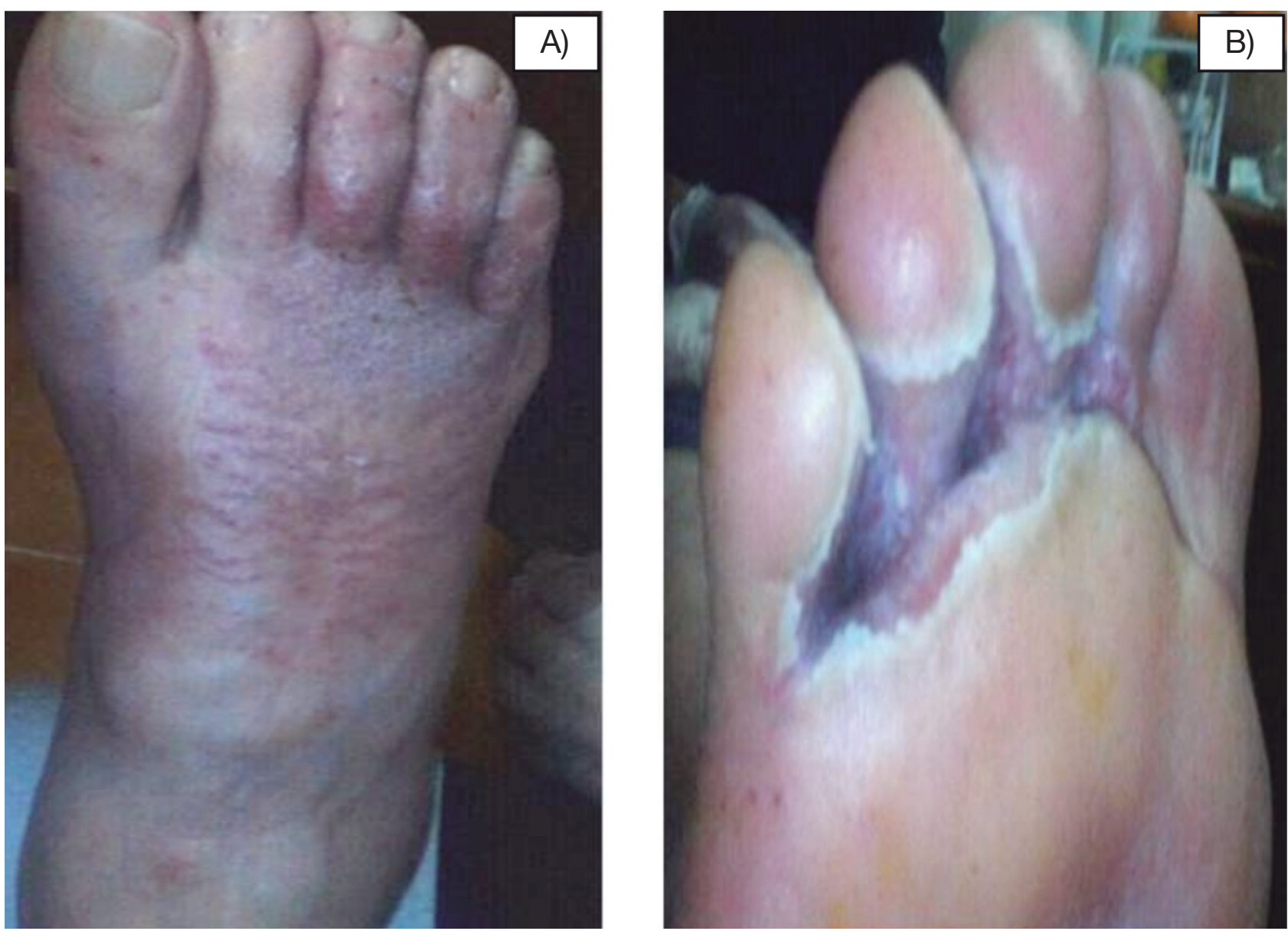

Figura 1. A) Dorso del pie con lesiones papulosas que se extienden desde los dedos hasta el tobillo. B) Región plantar del pie derecho con pérdida de tejido en la región interfalángica desde el segundo al quinto dedos.
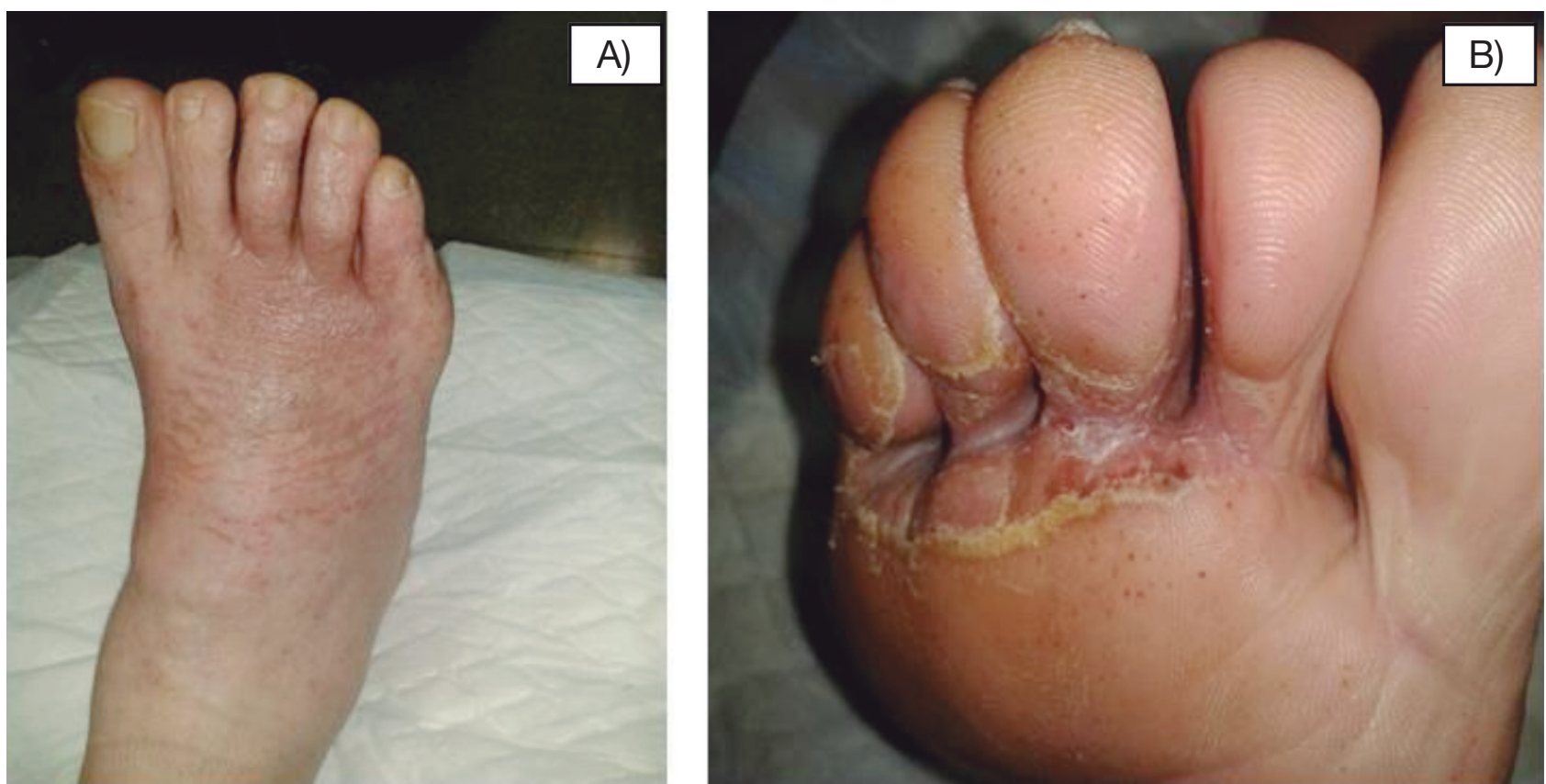

Figura 2. A) Dorso del pie con lesiones en remisión. B) Región plantar del pie derecho con lesión cicatricial en la región interfalángica desde el tercer al quinto dedo. 
bamos con el problema de la distancia al centro hospitalario desde el domicilio del paciente. Esto pudo solventarse gracias a la colaboración establecida entre el S.U.H. y el PAC del lugar donde residía el paciente. La administración de antibiótico intravenoso ambulatoria es una práctica que se empezó a realizar en los años 80 en nuestro país ${ }^{7}$ y que actualmente se lleva a cabo en las unidades de Hospitalización domiciliaria $(\mathrm{HaD})^{7-9}$. Estas unidades han disminuido costes casi en un $200 \%$ y libera camas hospitalarias, además de presentar ventajas para los pacientes ${ }^{7}$. En caso de la inexistencia de estas unidades, la posibilidad de utilizar los PAC o los Centros de Salud para la administración de tratamientos intravenosos en áreas con gran dispersión geográfica, facilitaría el acceso a tratamientos de muchos pacientes, además del ahorro que supondría.

\section{BIBLIOGRAFÍA}

1. Gary Sibbald R, Kelley J, Kennedy-Evans KL, Labrecque C, Waters N. A practical approach to the prevention and management of intertrigo, or moisture-associated skin da- mage, due to perspiration: expert consensus on best practice. Wound Care Canada-Supplement. 2013; 11 (2): 1-22.

2. Janniger CK, Schwartz RA, Szepietowski JC, Reich A. Intertrigo and common secondary skin infection. Am Fam Physician, 2005; 72: 833-8.

3. Wassilew SW. Infections of the skin caused by gram-negative pathogens. Foot infections-wound infection-folliculitis. Z Hautkr. 1989; 64 (1): 17-20.

4. Lin JY, Shih YL, Ho HC. Foot bacterial intertrigo mimicking interdigital tinea pedis. Chang Gung Med J. 2011; 34 (1): 44-9.

5. Talwar P, Kumar B, Ayyagiri A, Kaur S. Prevalence of bacteria and fungi in athlete's foot of varying severity and response to topical antibacterial and antifungal therapies. Sabouraudia. 1985; 23 (4): 303-12.

6. Wu DC, Chan WW, Metelitsa AI, Fiorillo L, Lin AN. Pseudomonas skin infection: clinical features, epidemiology, and management. Am J Clin Dermatol. 2011; 12 (3): 157-69.

7. González VJ, Bouza E. Tratamiento antimicrobiano intravenoso en el domicilio. Med Clin (Barc). 2008; 131 (8): 295-7.

8. Estrada O, Cuxart A, Bonet G. Tratamiento antibiótico por vía intravenosa domiciliario y hospitalización a domicilio. Med Clin (Barc). 2007; 128 (20): 798-9.

9. Laghzaoui F, Murcia J, Llorens P, Portillas J. Ceftazidima en infusión continua en el tratamiento de infecciones por Pseudomonas aeruginosa en una Unidad de Hospitalización a Domicilio. Enferm Infecc Microbiol Clin. 2007; 25 (1): 71-2. 